\title{
Theory of Electro Relativity
}

\author{
Greg Poole \\ Industrial Tests, Inc., Rocklin, CA, USA \\ Email: greg@indtest.com
}

How to cite this paper: Poole, G. (2019) Theory of Electro Relativity. Journal of High Energy Physics, Gravitation and Cosmology, 5, 1063-1067.

https://doi.org/10.4236/jhepgc.2019.54059

Received: July 28, 2019

Accepted: September 15, 2019

Published: September 18, 2019

Copyright $\odot 2019$ by author(s) and Scientific Research Publishing Inc. This work is licensed under the Creative Commons Attribution International License (CC BY 4.0).

http://creativecommons.org/licenses/by/4.0/

\begin{abstract}
A unified field theory is created by using a simple dipole antenna to model the Earth's electrical fields. From Maxwell's equation a set of standard radio field equations are presented for the Electrostatic, Inductive and Radiative field. By inserting three gravity field equations $\left(1 / r, 1 / r^{2}\right.$ and $\left.1 / r^{3}\right)$ a new set of field equations are created that can be used to define space, time, gravity and the electromagnetic field at any point $\mathrm{P}$ in the field.
\end{abstract}

\section{Keywords}

Dipole Antenna, Gravity Field, Maxwell's Equation, Unified Field Theory

\section{Introduction}

In this paper, I will use simple dipole radio equations, based on Maxwell equations, and insert my newly discovered gravity field equations for the $1 / r, 1 / r^{2}$ and $1 / r^{3}$ electromagnetic fields. By adding the contribution of each field, total gravity is achieved. The final series of equations will then include space, time, gravity and electromagnetism, which can be used to identify the variable at any point $\mathrm{P}$ in the fields. A simple antenna is thus used as a means to develop a unified field theory.

\section{History of Unified Field Theory}

In 1864, James Clerk Maxwell published his famous paper on a dynamical theory of the electromagnetic field. This was the first example of a theory that was able to encompass previously separate electrostatic and magnetic field theories to provide a unifying theory of electromagnetism. By 1905, Albert Einstein had used the constancy of the speed of light in Maxwell's theory to unify our notions of space and time into an entity we now call spacetime. In 1915, Einstein expanded this theory of special relativity to general relativity, using a gravity field to describe the curving geometry of four-dimensional spacetime. He created his gravity field from a centrifugal field using an equivalency principle (Figure 1). 


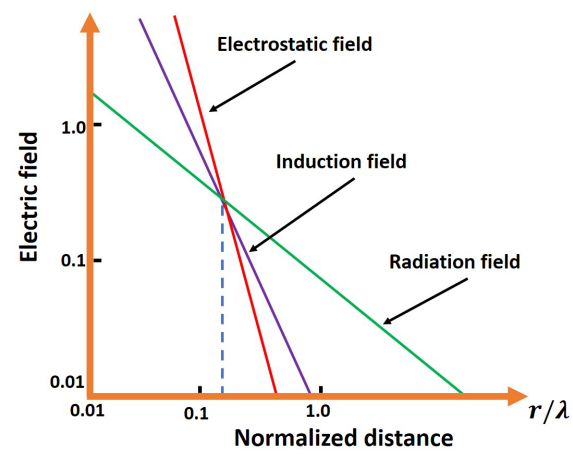

Figure 1. Radio fields \& gravity decay over distance.

The term Unified Field Theory was coined by Albert Einstein [1], who attempted to unify his general theory of relativity with electromagnetism. The "Theory of Everything" [2] and Grand Unified Theory [3] are closely related to unified field theory, but differ by not requiring the basis of nature to be fields, and often by attempting to explain physical constants of nature. Einstein relativity work is based on a unipolar (monopole) electrodynamic model, as opposed to my dipole model.

Following the creation of the general relativity theory, a large number of physicists and mathematicians attempted to unify the then-known fundamental interactions [4]. A large number of mathematicians and physicists, including Hermann Weyl, Arthur Eddington, and Theodor Kaluza also attempted to develop approaches that could unify these interactions [5] [6]. These scientists pursued several avenues of generalization, including extending the foundations of geometry and adding an extra spatial dimension.

In view of later developments in this domain, of particular interest are the theories of Hermann Weyl from 1919, who introduced the concept of an (electromagnetic) gauge field in a classical field theory [7] and, two years later, that of Theodor Kaluza, who extended General Relativity to five dimensions [8]. Continuing in this latter direction, Oscar Klein, in 1926, proposed that the fourth spatial dimension be curled up into a small, unobserved circle. In Kaluza-Klein theory, the gravitational curvature of the extra spatial direction behaves as an additional force similar to electromagnetism. These and other models of electromagnetism and gravity were pursued by Albert Einstein in his attempts at a classical unified field theory. By 1930, Einstein had already considered the Einstein-Maxwell-Dirac System.

\section{Gravity Field Equations}

Gravity Far Field:

$$
g_{F}=\frac{G c^{2}}{r}
$$

Or,

$$
c^{2} r=\frac{g_{F} r^{2}}{G}
$$


Gravity Near Field:

$$
g_{N}=\frac{G c^{3}}{2 \pi r^{2}}
$$

Or,

$$
c r^{2}=\frac{2 \pi g_{N} r^{4}}{G c^{2}}
$$

Gravity Magnetoquasistatic (Electrostatic) Field:

$$
g_{M}=\frac{G c^{4} \tau}{\pi r^{3}}
$$

where,

$$
\begin{gathered}
\tau=1 / f \\
\omega=2 \pi f=2 \pi / \tau
\end{gathered}
$$

Inserting, I obtain

$$
\begin{gathered}
\tau=\frac{g_{M} \pi r^{3}}{G c^{4}} \\
\frac{2 \pi}{\omega}=\frac{g_{M} \pi r^{3}}{G c^{4}} \\
\omega r^{3}=\frac{2 G c^{4}}{g_{M}}
\end{gathered}
$$

\section{Field Equations of an Antenna}

From Maxwell's equations the components of the fields at any point $\mathrm{P}$ are:

$$
\begin{aligned}
& H_{\phi}=I l \sin \theta\left[-\frac{\cos \omega\left(t-\frac{r}{c}\right)}{c} \frac{1}{r^{2}}+\frac{\omega \sin \omega\left(t-\frac{r}{c}\right)}{c^{2}} \frac{1}{r}\right] \\
& H_{r}=0 \\
& H_{\theta}=0 \\
& E_{\phi}=0 \\
& E_{r}=2 I l \cos \theta\left[\frac{\sin \omega\left(t-\frac{r}{c}\right)}{\omega} \frac{1}{r^{3}}+\frac{\cos \omega\left(t-\frac{r}{c}\right)}{c} \frac{1}{r^{2}}\right] \\
& E_{\theta}=I l \sin \theta\left[\frac{\sin \omega\left(t-\frac{r}{c}\right)}{\omega} \frac{1}{r^{3}}+\frac{\cos \omega\left(t-\frac{r}{c}\right)}{c} \frac{1}{r^{2}}+\frac{\omega \sin \omega\left(t-\frac{r}{c}\right)}{c^{2}} \frac{1}{r}\right]
\end{aligned}
$$

For $r \gg \lambda$ we have the radiation fields of the antenna: 


$$
\begin{gathered}
H_{\phi}=I l \sin \theta\left[\frac{\omega \sin \omega\left(t-\frac{r}{c}\right)}{c^{2}} \frac{1}{r}\right] \\
E_{r}=0 \\
E_{\theta}=I l \sin \theta\left[\frac{\omega \sin \omega\left(t-\frac{r}{c}\right)}{c^{2}} \frac{1}{r}\right]
\end{gathered}
$$

\section{Electro Relativity Equations}

$$
\begin{aligned}
& H_{\phi}=I l \sin \theta\left[-\frac{G c^{2} \cos \omega\left(t-\frac{r}{c}\right)}{2 \pi g_{N} r^{4}}+\frac{G \omega \sin \omega\left(t-\frac{r}{c}\right)}{g_{F} r^{2}}\right] \\
& H_{r}=0 \\
& H_{\theta}=0 \\
& E_{\phi}=0 \\
& E_{r}=2 I l \cos \theta\left[\frac{g_{M} \sin \omega\left(t-\frac{r}{c}\right)}{2 G c^{4}}+\frac{G c^{2} \cos \omega\left(t-\frac{r}{c}\right)}{2 \pi g_{N} r^{4}}\right] \\
& E_{\theta}=I l \sin \theta\left[\frac{g_{M} \sin \omega\left(t-\frac{r}{c}\right)}{2 G c^{4}}+\frac{G c^{2} \cos \omega\left(t-\frac{r}{c}\right)}{2 \pi g_{N} r^{4}}+\frac{G \omega \sin \omega\left(t-\frac{r}{c}\right)}{g_{F} r^{2}}\right]
\end{aligned}
$$

For $r \gg \lambda$ we have the radiation fields of the antenna:

$$
\begin{gathered}
H_{\phi}=I l \sin \theta\left[\frac{G \omega \sin \omega\left(t-\frac{r}{c}\right)}{g_{F} r^{2}}\right] \\
E_{r}=0 \\
E_{\theta}=I l \sin \theta\left[\frac{G \omega \sin \omega\left(t-\frac{r}{c}\right)}{g_{F} r^{2}}\right]
\end{gathered}
$$

\section{Conclusions}

Modeling of the Earth as a simple dipole antenna provides us with a view of the Earth's three-dimensional spherical electromagnetic field. Using the speed of light 
(c), the radius of the Earth (or field), Newton's Constant $(G)$ for proportionating and the flux transfer event frequency $(f)$, a new approach to gravity is explored using electromagnetism. By inserting three gravity field equations into standard radio equations a unified field theory is created that describes space, time, gravity and electromagnetism at any point $\mathrm{P}$ in the field. Gravity at any point $\mathrm{P}$ is determined by $g_{T}=g_{M}+g_{N}+g_{F}$.

We can readily conclude that maximum acceleration of light and compression of space in the magnetoquasistatic field give us a solid planet. And, atmospheric pressure of gases is derived from somewhat less acceleration and compression of space in the near field. We also know that atmospheric pressure has a mathematical relationship to gravity. No compression of space occurs in the far field.

\section{Acknowledgements}

The author wishes to acknowledge ASK Scientific for assistance in formatting the equations. The work of James Clerk Maxwell, Sir Joseph Larmor, Heinrick Hertz, Nickola Tesla and Guglielmo Marconi, the inventors of radio were instrumental in creating this electro relativity theory.

\section{Conflicts of Interest}

The author declares no conflicts of interest regarding the publication of this paper.

\section{References}

[1] How the Search for a Unified Theory Stumped Einstein to His Dying Day. phys.org.

[2] Hawking, S.W. (2006) The Theory of Everything: The Origin and Fate of the Universe. Phoenix Books.

[3] Ross, G. (1984) Grand Unified Theories. Westview Press, Boulder.

[4] Vizgin, V. (1994) Unified Field Theories in the First Third of the 20th Century. Birkhäuser, Basel.

[5] Weyl, H. (1918) Gravitation und Elektrizität. Sitzungsber. Preuss. Akad. Wiss., Berlin, 465 .

[6] Eddington, A.S. (1924) The Mathematical Theory of Relativity. 2nd Edition, Cambridge Univ. Press, Cambridge.

[7] Scholtz, E. (2001) Hermann Weyl's Raum-Zeit-Materie and a General Introduction to His Scientific Work. Birkhäuser, Basel. https://doi.org/10.1007/978-3-0348-8278-1

[8] Wuensch, D. (2003) The Fifth Dimension: Theodor Kaluza's Ground-Breaking Idea. Annalen der Physik, 12, 519-542. https://doi.org/10.1002/andp.200310025

[9] Electrical Engineering of the Westinghouse Electric Corporation (1948) Industrial Electronics Reference Book. Chapter 20, John Wiley \& Sons, Inc., Hoboken, 308-310. 